\title{
Inner Cauchy Horizon of Axisymmetric and Stationary Black Holes with Surrounding Matter in Einstein-Maxwell Theory
}

\author{
Marcus Ansorg* and Jörg Hennig ${ }^{\dagger}$ \\ Max Planck Institute for Gravitational Physics, Am Mühlenberg 1, D-14476 Golm, Germany
}

(Received 31 March 2009; published 5 June 2009)

\begin{abstract}
We study the interior electrovacuum region of axisymmetric and stationary black holes with surrounding matter and find that there exists always a regular inner Cauchy horizon inside the black hole, provided the angular momentum $J$ and charge $Q$ of the black hole do not vanish simultaneously. In particular, we derive an explicit relation for the metric on the Cauchy horizon in terms of that on the event horizon. Moreover, our analysis reveals the remarkable universal relation $(8 \pi J)^{2}+\left(4 \pi Q^{2}\right)^{2}=A^{+} A^{-}$, where $A^{+}$ and $A^{-}$denote the areas of event and Cauchy horizon, respectively.
\end{abstract}

DOI: 10.1103/PhysRevLett.102.221102

PACS numbers: 04.70.Bw, 04.20.Cv, 04.40.Nr

Introduction.-In the interior of a single rotating, electrically charged, axisymmetric, and stationary black hole in electrovacuum (described by the Kerr-Newman family of solutions) there exists a Cauchy horizon $\mathcal{H}^{-}$, which is the future boundary of the domain of dependence of the event horizon $\mathcal{H}^{+}$. The presence of this Cauchy horizon is related to the fact that, in Boyer-Lindquist coordinates, the axisymmetric and stationary Einstein-Maxwell vacuum equations are hyperbolic within an interior vicinity of $\mathcal{H}^{+}$. The two horizons $\mathcal{H}^{+}$and $\mathcal{H}^{-}$represent the past and future boundary of this hyperbolic region. Interestingly, for the Kerr-Newman family the areas $A^{ \pm}$of the horizons $\mathcal{H}^{ \pm}$are related by

$$
(8 \pi J)^{2}+\left(4 \pi Q^{2}\right)^{2}=A^{+} A^{-},
$$

where $J$ and $Q$ are the angular momentum and charge of the black hole, respectively.

In pure Einsteinian gravity (i.e., without Maxwell field), these observations have been generalized in [1]. It was shown that for axisymmetric and stationary black holes with surrounding matter there always exists a regular inner Cauchy horizon, provided that $J \neq 0$ holds. It was also shown that such black holes satisfy relation (1) (with $Q=0)$ in general.

In this Letter, we investigate axisymmetric and stationary black holes with surrounding matter and include electromagnetic fields. For our analysis, we will make use of a linear matrix problem, whose integrability conditions are equivalent to the Einstein-Maxwell equations in vacuum (see [2]). The existence of such a linear problem (LP) permits the application of so-called soliton methods (e.g. the "inverse scattering method") through which particular solutions of the field equations in question can be found (see, e.g., $[3,4]$ ).

Here we integrate the LP along the boundaries of the hyperbolic region inside the black hole and obtain in this manner useful relations between the field quantities at these boundaries. In particular, we are able to calculate the metric and electromagnetic potentials on $\mathcal{H}^{-}$in terms of those on $\mathcal{H}^{+}$. Moreover, we find that from these relations Eq. (1) can be deduced; i.e., Eq. (1) turns out to be valid for arbitrary axisymmetric and stationary black holes with surrounding matter in Einstein-Maxwell theory.

A detailed description of the calculations sketched below will be given in a forthcoming paper.

Coordinate systems and Einstein-Maxwell equations.In the following, we study an electrovacuum vicinity of the black hole's event horizon. (Note that for a stationary spacetime, the immediate vicinity of a black hole event horizon must be electrovacuum; see [5,6].) In this vicinity, we introduce Weyl coordinates $(\varrho, \zeta, \varphi, t)$ in which the line element reads as follows

$$
\begin{aligned}
d s^{2}= & e^{-2 U}\left[e^{2 k}\left(d \varrho^{2}+d \zeta^{2}\right)+\varrho^{2} d \varphi^{2}\right] \\
& -e^{2 U}(d t+a d \varphi)^{2}
\end{aligned}
$$

where the metric potentials $U, k$, and $a$ are functions of $\varrho$ and $\zeta$ alone. As sketched in Fig. 1 (left panel), the event horizon $\mathcal{H}^{+}$is located on the interval $-2 r_{h} \leq \zeta \leq 2 r_{h}$, $r_{h}=$ const, of the $\zeta$-axis. The remaining part $|\zeta|>2 r_{h}$ of the $\zeta$-axis corresponds to the rotation axis.
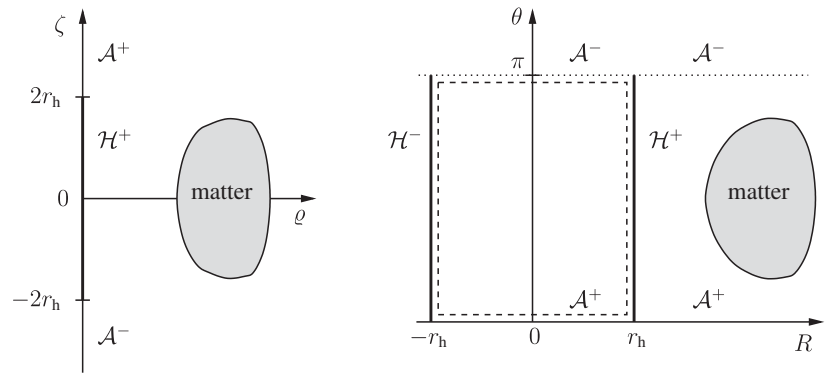

FIG. 1. Sketch of a part of a black hole spacetime in Weyl coordinates (left panel) and Boyer-Lindquist type coordinates (right panel). $\mathcal{A}^{+}$and $\mathcal{A}^{-}$denote upper and lower parts of the symmetry axis and $\mathcal{H}^{+}$and $\mathcal{H}^{-}$denote event and Cauchy horizons. In the Letter we will integrate the linear problem for the Einstein-Maxwell equations along the closed dashed line. 
In order to investigate the interior of the black hole, which is characterized by negative values of $\varrho^{2}$, we also introduce Boyer-Lindquist-type coordinates $(R, \theta, \varphi, t)$ via

$$
\varrho^{2}=4\left(R^{2}-r_{h}^{2}\right) \sin ^{2} \theta, \quad \zeta=2 R \cos \theta .
$$

In these coordinates, the event horizon $\mathcal{H}^{+}$and the inner Cauchy horizon $\mathcal{H}^{-}$are located at $R=r_{h}$ and $R=-r_{h}$ respectively; see Fig. 1 (right panel).

In the electrovacuum region, the electromagnetic field alone constitutes the energy momentum tensor

$$
T_{i j}=\frac{1}{4 \pi}\left(F_{k i} F_{j}^{k}-\frac{1}{4} g_{i j} F_{k l} F^{k l}\right),
$$

where $F_{i j}$ is the electromagnetic field tensor. We use the Lorenz gauge, in which $F_{i j}$ can be written in terms of a vector potential $\left(A_{i}\right)=\left(0,0, A_{\varphi}, A_{t}\right)$,

$$
F_{i j}=A_{i, j}-A_{j, i}
$$

Note that, like the metric quantities, $A_{\varphi}$ and $A_{t}$ also depend on $R$ and $\theta$ only.

We introduce the complex electromagnetic potential $\Phi$ and the complex Ernst potential $\mathcal{E}[7,8]$ by

$$
\Phi=A_{t}+i \beta, \quad \mathcal{E}=e^{2 U}-|\Phi|^{2}+i b,
$$

where the imaginary parts $b$ and $\beta$ are related to metric and vector potentials via

$$
\begin{aligned}
a_{, \varrho} & =\varrho e^{-4 U}\left[b_{, \zeta}-i\left(\bar{\Phi} \Phi_{, \zeta}-\Phi \bar{\Phi}_{, \zeta}\right)\right], \\
a_{, \zeta} & =-\varrho e^{-4 U}\left[b_{, \varrho}-i\left(\bar{\Phi} \Phi_{, \varrho}-\Phi \bar{\Phi}_{, \varrho}\right)\right], \\
\beta_{, \varrho} & =\frac{e^{2 U}}{\varrho}\left(a A_{t, \zeta}-A_{\varphi, \zeta}\right), \\
\beta_{, \zeta} & =-\frac{e^{2 U}}{\varrho}\left(a A_{t, \varrho}-A_{\varphi, \varrho}\right) .
\end{aligned}
$$

In this formulation (the bar denotes complex conjugation), the Einstein-Maxwell equations in electrovacuum are equivalent to the two complex Ernst equations [7]

$$
\begin{aligned}
e^{2 U} \Delta \mathcal{E} & =\nabla \mathcal{E} \cdot(\nabla \mathcal{E}+2 \bar{\Phi} \nabla \Phi), \\
e^{2 U} \Delta \Phi & =\nabla \Phi \cdot(\nabla \mathcal{E}+2 \bar{\Phi} \nabla \Phi) .
\end{aligned}
$$

Here, $\Delta$ and $\nabla$ denote Laplace and nabla operators in flat cylindrical coordinates $(\varrho, \zeta, \varphi)$.

The linear problem.-As mentioned in the introduction, the existence of the LP, whose integrability conditions are equivalent to (8), is crucial for our analysis. For its formulation we introduce the complex coordinates $z=\varrho+$ $i \zeta, \bar{z}=\varrho-i \zeta$ and the function

$$
\lambda(K, z, \bar{z})=\sqrt{\frac{K-i \bar{z}}{K+i z}}
$$

which depends on a spectral parameter $K \in \mathbb{C}$. For fixed values $z, \bar{z}$, Eq. (9) describes a spectral mapping $\mathbb{C} \rightarrow \mathbb{C}$, $K \mapsto \lambda$ from a two-sheeted Riemann surface ( $K$-plane) onto the complex $\lambda$-plane. The two $K$ sheets are connected at the two branch points $K_{1}=i \bar{z}(\lambda=0)$ and $K_{2}=-i z$ $(\lambda=\infty)$.

The LP is a system of first order equations for a $3 \times 3$ matrix pseudopotential $\boldsymbol{\Omega}=\boldsymbol{\Omega}(K, z, \bar{z})$, which reads [2]

$$
\begin{aligned}
& \boldsymbol{\Omega}_{, z}=\left[\left(\begin{array}{ccc}
B_{1} & 0 & E_{1} \\
0 & A_{1} & 0 \\
-F_{1} & 0 & \frac{1}{2}\left(A_{1}+B_{1}\right)
\end{array}\right)+\lambda\left(\begin{array}{ccc}
0 & B_{1} & 0 \\
A_{1} & 0 & -E_{1} \\
0 & -F_{1} & 0
\end{array}\right)\right] \boldsymbol{\Omega}, \\
& \boldsymbol{\Omega}_{, \bar{z}}=\left[\left(\begin{array}{ccc}
B_{2} & 0 & E_{2} \\
0 & A_{2} & 0 \\
-F_{2} & 0 & \frac{1}{2}\left(A_{2}+B_{2}\right)
\end{array}\right)+\frac{1}{\lambda}\left(\begin{array}{ccc}
0 & B_{2} & 0 \\
A_{2} & 0 & -E_{2} \\
0 & -F_{2} & 0
\end{array}\right)\right] \boldsymbol{\Omega},
\end{aligned}
$$

where

$$
\begin{array}{ll}
A_{1}=\frac{1}{2} e^{-2 U}\left(\mathcal{E}_{, z}+2 \bar{\Phi} \Phi_{, z}\right), & E_{1}=i e^{-U} \Phi_{, z}, \\
B_{1}=\frac{1}{2} e^{-2 U}\left(\overline{\mathcal{E}}_{, z}+2 \Phi \bar{\Phi}_{, z}\right), & F_{1}=i e^{-U} \bar{\Phi}_{, z} .
\end{array}
$$

Analogous expressions for $A_{2}, B_{2}, E_{2}$, and $F_{2}$ can be obtained from (11) by replacing $z$ with $\bar{z}$.

If $\boldsymbol{\Omega}$ is a solution of the LP (10), then $\boldsymbol{\Omega C}(K)$ is also a solution for every $3 \times 3$ matrix function $\mathbf{C}(K)$. We can always find a $\mathbf{C}(K)$ to bring $\boldsymbol{\Omega}$ into the form

$$
\boldsymbol{\Omega}^{>}(K, z, \bar{z})=\left(\begin{array}{ccc}
\psi_{1}^{>}(K, z, \bar{z}) & \psi_{1}^{<}(K, z, \bar{z}) & 0 \\
\psi_{2}^{>}(K, z, \bar{z}) & -\psi_{2}^{<}(K, z, \bar{z}) & 0 \\
\psi_{3}^{>}(K, z, \bar{z}) & \psi_{3}^{<}(K, z, \bar{z}) & 0
\end{array}\right),
$$

which depends on three functions $\psi_{1}, \psi_{2}, \psi_{3}$. Here, the superscripts " $>$ " or " $<$ " indicate whether the functions are evaluated in the upper $(\lambda=1$ for $K=\infty)$ or lower $(\lambda=-1$ for $K=\infty)$ sheet of the two-sheeted Riemann $K$ surface. By interchanging " $>$ " and " $<$ " in (12), we obtain a similar equation for $\boldsymbol{\Omega}<$. Obviously, $\boldsymbol{\Omega}$ in the form (12) is not invertible. Nevertheless, we will see that it still contains sufficient information about $\mathcal{E}$ and $\Phi$. 
For our analysis of the LP it is also useful to study the situation in a frame of reference that rotates with a constant angular velocity $\omega_{0}$ with respect to our original frame. In this coordinate system $\left(\varrho, \zeta, \varphi^{\prime}, t\right)$, the only new coordinate reads $\varphi^{\prime}=\varphi-\omega_{0} t$. Note that line element, Ernst equations and LP preserve their form in this rotating frame. The transformed pseudopotential $\boldsymbol{\Omega}^{\prime}$ can be obtained from $\boldsymbol{\Omega}$ via

$$
\boldsymbol{\Omega}^{\prime}=\left[\left(\begin{array}{ccc}
c_{-} & 0 & 0 \\
0 & c_{+} & 0 \\
0 & 0 & \sqrt{c_{+} c_{-}}
\end{array}\right)+\alpha\left(\begin{array}{ccc}
-1 & -\lambda & 0 \\
\lambda & 1 & 0 \\
0 & 0 & 0
\end{array}\right)\right] \boldsymbol{\Omega}
$$

with

$$
c_{ \pm}:=1+\omega_{0}\left(a \pm \varrho e^{-2 U}\right), \quad \alpha:=i(K+i z) \omega_{0} e^{-2 U} .
$$

(cf. [4,9] for the corresponding transformation valid in pure gravity, i.e., without electromagnetic field.)

Solution of the linear problem.-We are able to integrate the LP along the dashed line in Fig. 1 (right panel) since the metric inside the entire hyperbolic region (excluding $\mathcal{H}^{-}$ for the time being) is regular. This regularity is a consequence of the requirement that the metric potentials be analytic functions of $R$ and $\cos \theta$ in an exterior vicinity of $\mathcal{H}^{+}$, including $\mathcal{H}^{+}$, see theorem 6.3 in [10]. Note that with the arguments presented there, this theorem can be carried over to the Einstein-Maxwell case considered here [11].

On the entire integration path, the Weyl coordinate $\varrho$ vanishes, see (3), leading to $\lambda= \pm 1$. For $\varrho=0$ and $\lambda=1$ the LP reduces to an ODE with the general solution

$$
\boldsymbol{\Omega}=\left(\begin{array}{ccc}
\overline{\mathcal{E}}+2|\Phi|^{2} & 1 & \Phi \\
\mathcal{E} & -1 & -\Phi \\
-2 i e^{U} \bar{\Phi} & 0 & -i e^{U}
\end{array}\right) \mathbf{C}(K) .
$$

Here, $\mathbf{C}$ is a $3 \times 3$ matrix that depends on $K$ only. Respecting the gauge (12), the third column of $\mathbf{C}$ vanishes.

On all four parts of our integration path, $\boldsymbol{\Omega}$ has the form (14), but with different "integration constants" C. We denote these with $\mathbf{C}$ on $\mathcal{A}^{+}, \widetilde{\mathbf{C}}$ on $\mathcal{A}^{-}, \mathbf{D}$ on $\mathcal{H}^{+}$, and $\tilde{\mathbf{D}}$ on $\mathcal{H}^{-}$. Moreover, we can normalize $\boldsymbol{\Omega}$ such that

$$
\mathbf{C}(K)=\left(\begin{array}{ccc}
C_{1}(K) & 0 & 0 \\
C_{2}(K) & \psi(K) & 0 \\
C_{3}(K) & 0 & 0
\end{array}\right), \quad \psi:=\left(K^{2}-4 r_{h}^{2}\right)^{3},
$$

holds for the integration constant on $\mathcal{A}^{+}$.

From (14), we also calculate the pseudopotentials in the two different rotating frames of reference with $\omega_{0}=\omega^{ \pm}$ cf. (13), where $\omega^{ \pm}=\left.\omega\right|_{R= \pm r_{h}}=$ const denotes the angular velocities of the horizons $\mathcal{H}^{ \pm}$. In particular, $\omega^{ \pm}$and the metric potential $a$ are related by the horizon boundary conditions $a=-1 / \omega^{ \pm}$valid on $\mathcal{H}^{ \pm}$. Note that $a=0$ on $\mathcal{A}^{ \pm}$. Now, the pseudopotentials $\boldsymbol{\Omega}$ and $\boldsymbol{\Omega}^{\prime}$ (in both rotating frames of reference) are continuous at the north and south poles of the horizons $\mathcal{H}^{ \pm}$. This leads us to an algebraic system of equations for the elements of $\tilde{\mathbf{C}}, \mathbf{D}$, and $\tilde{\mathbf{D}}$ in terms of $\mathbf{C}$. From the solution of this system we are able to derive the solution of the LP on the entire integration path, expressed in terms of the three functions $C_{1}(K), C_{2}(K)$, and $C_{3}(K)$.

Potentials on the Cauchy horizon.-From the pseudopotential $\boldsymbol{\Omega}$, we now calculate the potentials $\mathcal{E}$ and $\Phi$ on $\mathcal{H}^{-}$. In a first step, we express $C_{1}, C_{2}$, and $C_{3}$ in terms of the event horizon potentials.

At the branch points $K_{1}=i \bar{z}$ and $K_{2}=-i z, \mathbf{\Omega}$ is unique; i.e., the values in both $K$ sheets coincide. In particular, for $\varrho=0$ (where $K_{1}=K_{2}=\zeta$ ) we have

$$
\psi_{i}^{>}=\psi_{i}^{<}, \quad i=1,2,3, \quad \text { for } K=\zeta .
$$

Considering these conditions at $\mathcal{H}^{+}$, it follows that

$$
\begin{aligned}
C_{1}(\zeta)= & n\left[\overline{\mathcal{E}}+2 \Phi_{N}^{+} \bar{\Phi}-2 i \omega^{+}\left(\zeta-2 r_{h}\right)+\mathcal{E}_{N}^{+}\right], \\
C_{2}(\zeta)= & \psi+n\left[\left(\mathcal{E}_{N}^{+}+2\left|\Phi_{N}^{+}\right|^{2}\right)\left(\overline{\mathcal{E}}+2 \Phi_{N}^{+} \bar{\Phi}+\mathcal{E}_{N}^{+}\right)\right. \\
& \left.+2 i \omega^{+}\left(\zeta-2 r_{h}\right) \overline{\mathcal{E}}\right], \\
C_{3}(\zeta)= & -2 n\left[\bar{\Phi}_{N}^{+}\left(\overline{\mathcal{E}}+2 \Phi_{N}^{+} \bar{\Phi}+\mathcal{E}_{N}^{+}\right)\right. \\
& \left.-2 i \omega^{+}\left(\zeta-2 r_{h}\right) \bar{\Phi}\right],
\end{aligned}
$$

where $\mathcal{E}$ and $\Phi$ denote the potentials on $\mathcal{H}^{+}$and with

$$
n:=\frac{\mathcal{E}+2 \bar{\Phi}_{N}^{+} \Phi+2 i \omega^{+}\left(\zeta-2 r_{h}\right)-\mathcal{E}_{N}^{+}-2\left|\Phi_{N}^{+}\right|^{2}}{4\left(\omega^{+}\right)^{2}\left(\zeta-2 r_{h}\right)^{2} e^{2 U}} \psi .
$$

Here, we have introduced the notations $(\cdot)_{N}^{ \pm}$and $(\cdot)_{S}^{ \pm}$for values at the north pole $(\theta=0)$ and the south pole $(\theta=\pi)$ of the horizons $\mathcal{H}^{ \pm}\left(R= \pm r_{h}\right)$, respectively.

Now, we evaluate (16) on $\mathcal{H}^{-}$and solve the resulting conditions for $\mathcal{E}$ and $\Phi$. In terms of the Boyer-Lindquisttype coordinate $\theta$, we obtain the remarkable explicit relations

$$
\begin{aligned}
\mathcal{E}^{-}(\theta) & =\frac{a_{1}(\theta) \mathcal{E}^{+}(\pi-\theta)+a_{2}(\theta) \Phi^{+}(\pi-\theta)+a_{3}(\theta)}{c_{1}(\theta) \mathcal{E}^{+}(\pi-\theta)+c_{2}(\theta) \Phi^{+}(\pi-\theta)+c_{3}(\theta)}, \\
\Phi^{-}(\theta) & =\frac{b_{1}(\theta) \mathcal{E}^{+}(\pi-\theta)+b_{2}(\theta) \Phi^{+}(\pi-\theta)+b_{3}(\theta)}{c_{1}(\theta) \mathcal{E}^{+}(\pi-\theta)+c_{2}(\theta) \Phi^{+}(\pi-\theta)+c_{3}(\theta)},
\end{aligned}
$$

between the potentials on $\mathcal{H}^{-}$(superscript "-") and on $\mathcal{H}^{+}$(superscript "+"). The functions $a_{i}, b_{i}, c_{i}, i=1,2$, 3 , are specific polynomials in $\cos \theta$ which will be given explicitly in [12].

A thorough discussion of (19) reveals that the regularity of the potentials on $\mathcal{H}^{+}$implies that of the potentials on $\mathcal{H}^{-}$, provided that $J$ and $Q$ do not both vanish. In the limit of vanishing $J$ and $Q$, the potentials $\mathcal{E}^{-}$and $\Phi^{-}$diverge.

A universal equality. - In this section, we derive that (1) is true for arbitrary axisymmetric and stationary black holes with surrounding matter in Einstein-Maxwell theory. As in pure Einsteinian gravity (cf. [1]) it is possible to express $J, Q$, and $A^{ \pm}$in terms of potential values at north 
and south poles of the horizons $\mathcal{H}^{ \pm}$:

$$
\begin{aligned}
& J=\frac{1}{8\left(\omega^{+}\right)^{2}}\left[b_{S}^{+}-b_{N}^{+}-8 \omega^{+} r_{h}+2 A_{t}^{+}\left(\beta_{S}^{+}-\beta_{N}^{+}\right)\right], \\
& Q=\frac{1}{2 \omega^{+}}\left(\beta_{N}^{+}-\beta_{S}^{+}\right), \quad A^{ \pm}= \pm \frac{32 \pi r_{h}}{\left.e^{2 U}{ }_{, R}\right|_{N} ^{ \pm}},
\end{aligned}
$$

with $\omega^{+}=\frac{1}{4}\left[b_{, R}+2\left(A_{t} \beta_{, R}-\beta A_{t, R}\right)\right]_{N}^{+}$.

In order to calculate $e^{2 U}{ }_{, R} \mid \frac{ \pm}{N}$, we use the solution of the LP on $\mathcal{A}^{+}$. Evaluation of the conditions (16) leads us to the simple relation

$$
e^{2 U}=\frac{\psi(\zeta)}{C_{1}(\zeta)} \quad \text { on } \mathcal{A}^{+} .
$$

The explicit expression (17a) for $C_{1}(\zeta)$ and the potential horizon boundary conditions at $\mathcal{H}^{+}$, together with a careful study of the Einstein-Maxwell equations at the north and south pole of $\mathcal{H}^{+}$, yields

$$
\begin{aligned}
A^{+}= & -\left.\frac{2 \pi}{\left(\omega^{+}\right)^{2}} e^{2 U}{ }_{, \theta \theta}\right|_{N} ^{+} \\
A^{-}= & -\frac{\pi}{\left.2\left(\omega^{+}\right)^{2} e^{2 U}{ }_{, \theta \theta}\right|_{N} ^{+}}\left[\left(\beta_{N}^{+}-\beta_{S}^{+}\right)^{4}\right. \\
& \left.+\left(b_{S}^{+}-b_{N}^{+}-8 \omega^{+} r_{h}+2 A_{t}^{+}\left(\beta_{S}^{+}-\beta_{N}^{+}\right)\right)^{2}\right] .
\end{aligned}
$$

Hence with the above expressions for $J, Q$ and $A^{ \pm}$we conclude that Eq. (1) is satisfied.

Finally, combining our results with a closely related inequality obtained in [13], we arrive at the following.

Theorem. 1.-Every regular axisymmetric and stationary Einstein-Maxwell black hole with surrounding matter has a regular inner Cauchy horizon if and only if the angular momentum $J$ and charge $Q$ do not both vanish. Then the universal relation

$$
(8 \pi J)^{2}+\left(4 \pi Q^{2}\right)^{2}=A^{+} A^{-}
$$

is satisfied, where $A^{+}$and $A^{-}$denote the areas of event and inner Cauchy horizon, respectively. If, in addition, the black hole is subextremal (i.e., if there exist trapped surfaces in every sufficiently small interior vicinity of the event horizon), then the following inequalities hold:

$$
A^{-}<\sqrt{(8 \pi J)^{2}+\left(4 \pi Q^{2}\right)^{2}}<A^{+} .
$$

We would like to thank Gernot Neugebauer and Piotr T. Chruściel for many valuable discussions. This work was supported by the Deutsche Forschungsgemeinschaft (DFG) through the Collaborative Research Centre SFB/ TR7 "Gravitational wave astronomy."

*mans@aei.mpg.de

†pjh@aei.mpg.de

[1] M. Ansorg and J. Hennig, Classical Quantum Gravity 25, 222001 (2008).

[2] G. Neugebauer and D. Kramer, J. Phys. A 16, 1927 (1983).

[3] G. Neugebauer and R. Meinel, Phys. Rev. Lett. 73, 2166 (1994); 75, 3046 (1995).

[4] G. Neugebauer and R. Meinel, J. Math. Phys. (N.Y.) 44, 3407 (2003)

[5] J.M. Bardeen, in Black holes (Les Houches), edited by C. deWitt and B. deWitt (Gordon and Breach, London, 1973), p. 241.

[6] B. Carter, in Black Holes (Les Houches), edited by C. deWitt and B. deWitt (Gordon and Breach, London, 1973), p. 57.

[7] F. J. Ernst, Phys. Rev. 168, 1415 (1968).

[8] H. Stephani, D. Kramer, M. MacCallum, C. Hoenselaers, and E. Herlt, Exact Solutions of Einstein's Field Equations (University Press, Cambridge, 2003).

[9] G. Neugebauer, Ann. Phys. (Leipzig) 9, 342 (2000).

[10] P. T. Chruściel, Ann. Phys. (N.Y.) 202, 100 (1990).

[11] P. T. Chruściel (private communication).

[12] J. Hennig and M. Ansorg, arXiv:0904.2071.

[13] J. Hennig, C. Cederbaum, and M. Ansorg, arXiv:0812.2811. 